Corresponding author: peterson.jess@mayo.edu

(c) 2019 Peterson et al. This article is distributed under the terms of the Creative Commons Attribution-NonCommercial

License, which permits reuse and redistribution, except for commercial purposes, provided that the original author and source are credited.

Ontology term: B-cell lymphoma

Published by Cold Spring Harbor Laboratory Press

doi:10.1101/mcs.a004077

\section{Elucidating a false-negative MYC break- apart fluorescence in situ hybridization probe study by next-generation sequencing in a patient with high-grade B-cell lymphoma with IGH/MYC and IGH/BCL2 rearrangements}

\author{
Jess F. Peterson, ${ }^{1,4}$ Beth A. Pitel, ${ }^{1,4}$ Stephanie A. Smoley, ${ }^{1}$ George Vasmatzis, ${ }^{2}$ \\ James B. Smadbeck, ${ }^{2}$ Patricia T. Greipp, ${ }^{1}$ Rhett P. Ketterling, ${ }^{1,3}$ William R. Macon, ${ }^{3}$ \\ and Linda B. Baughn ${ }^{1}$ \\ ${ }^{1}$ Division of Laboratory Genetics and Genomics, Department of Laboratory Medicine and Pathology, ${ }^{2}$ Center \\ for Individualized Medicine-Biomarker Discovery, ${ }^{3}$ Division of Hematopathology, Department of Laboratory \\ Medicine and Pathology, Mayo Clinic, Rochester, Minnesota 55905, USA
}

Abstract The identification of MYC rearrangements in several mature B-cell neoplasms is critical for diagnostic and prognostic purposes. Commercially available fluorescence in situ hybridization (FISH) probe sets, including IGH/MYC dual-color dual-fusion (D-FISH) and MYC break-apart probes (BAPs), serve as the primary methodology utilized to detect MYC rearrangements. However, performing either IGH/MYC D-FISH or MYC BAP FISH studies in isolation has been reported to result in false-negative results because of the complex nature of $8 q 24$ rearrangements involving the MYC gene region. We report a 60-yr-old male with newly diagnosed high-grade B-cell lymphoma with a negative MYC BAP study, but with positive $B C L 2$ and $B C L 6 B A P$ studies. Per our current laboratory algorithm to concurrently interrogate the MYC gene region with both MYCBAP and IGH/MYCD-FISH probe sets, we performed IGH/MYC D-FISH studies and detected an IGH/MYC fusion. To further characterize the discrepant MYC results obtained by FISH, a next-generation sequencing strategy, mate-pair sequencing (MPseq), was performed and revealed a small insertion ( 200 kb) of the IGH locus downstream from the MYC gene that was undetectable by MYC BAP studies. This case highlights the importance of utilizing both IGH/MYC D-FISH and MYCBAP sets to detect potential cryptic MYC rearrangements and also demonstrates the power of MPseq to characterize complex structural rearrangements and copy-number abnormalities unappreciable by FISH.

\section{INTRODUCTION}

High-grade B-cell lymphoma (HGBL) with MYC and BCL2 and/or BCL6 rearrangements (previously referred to as double- or triple-hit lymphoma) is a highly aggressive mature B-cell neoplasm most often observed in elderly patients (Kluin et al. 2017; Li et al. 2012, 2016;

\footnotetext{
${ }^{4}$ These authors contributed equally to this work.
} 
Snuderl et al. 2010; Wang et al. 2015). The detection of MYC rearrangements in HGBL and other mature B-cell lymphomas has traditionally relied upon fluorescence in situ hybridization (FISH) strategies (dual-color dual-fusion [D-FISH] and/or break-apart probes [BAP]) that are typically performed on formalin-fixed paraffin-embedded (FFPE) tissue specimens. Although the most common MYC translocation partner is the immunoglobulin heavy chain (IGH) gene, which can usually be detected by IGH/MYCD-FISH studies, the MYCBAP has an added value for the detection of IGK/MYC, IGL/MYC, and non-IG/MYC rearrangements (Muñoz-Mármol et al. 2013; Sesques and Johnson 2017). Importantly, several studies have demonstrated that performing either IGH/MYC D-FISH or MYC BAP studies in isolation can result in false-negative results because of the broad range of $8 q 24$ breakpoints that occur in close proximity to the MYC gene region (May et al. 2010; Sun et al. 2012; Muñoz-Mármol et al. 2013; King et al. 2018).

Until recently, the characterization of cryptic IGH/MYC fusions has been limited to commercially available FISH probe sets (May et al. 2010; Sun et al. 2012; Muñoz-Mármol et al. 2013). Utilizing mate-pair sequencing (MPseq), a next-generation sequencing (NGS) technology that can detect both balanced and unbalanced structural and copy-number abnormalities, our group previously characterized four unique IGH/MYC rearrangement mechanisms observed in mature B-cell neoplasms (Drucker et al. 2014; Johnson et al. 2018; King et al. 2018; Smadbeck et al. 2018). Expanding on our previous report, we describe a 60-yr-old male with newly diagnosed HGBL with negative MYC BAP studies and a novel, cryptic IGH/MYC rearrangement characterized by MPseq.

\section{RESULTS}

\section{Hematopathology Evaluation}

The patient is a 60-yr-old male with a reported long-standing history of low-grade follicular lymphoma. Stained slides and FFPE blocks of a left iliac lymph node were submitted for tumor characterization. The lymph node architecture was effaced by a diffuse infiltrate of neoplastic large cells with plasmablastic cytologic features. There was extensive necrosis, and viable-appearing tumor cells were often perivascular in distribution within these areas.

By flow-cytometric immunophenotyping, $61 \%$ of the total analyzed events and $93 \%$ of the gated lymphoid events consisted of CD19 (dim) and cytoplasmic CD22-positive B cells that had partial coexpression of $C D 10$ and were immunoglobulin light-chain indeterminate. These $B$ cells were negative for CD20, CD5, CD23, CD34, cytoplasmic CD79a, and terminal deoxynucleotidyl transferase (TdT).

Immunoperoxidase studies were performed using antibodies directed against the following antigens: CD3, CD10, CD20, CD21, CD138, BCL2, BCL6, $\kappa$ and $\lambda$ immunoglobulin light chains, MUM1, MYC, and PAX5. The large, neoplastic lymphocytes with plasmablastic cytologic features were CD138 and MUM1-positive, showed expression of CD10 (partial), MYC (90\% positive), and BCL2 (100\% positive), and contained monotypic cytoplasmic $\kappa$ immunoglobulin light chains. The $B$ cells were negative for all other antigens for which testing was performed, including CD20, PAX5, and BCL6. No CD21-staining follicular dendritic cell networks were seen.

\section{Genomic Analyses}

All genomic studies were performed on a freshly submitted left iliac lymph node specimen. FISH performed on the FFPE lymph node specimen was negative for MYC rearrangement by MYCBAP studies (Fig. 1A) and positive for BCL2 and BCL6 rearrangements in $100 \%$ of interphase nuclei analyzed (abnormal cutoffs: $\geq 7 \%, \geq 9 \%$, and $\geq 6 \%$, respectively) (Fig. $1 C, D$ ). 

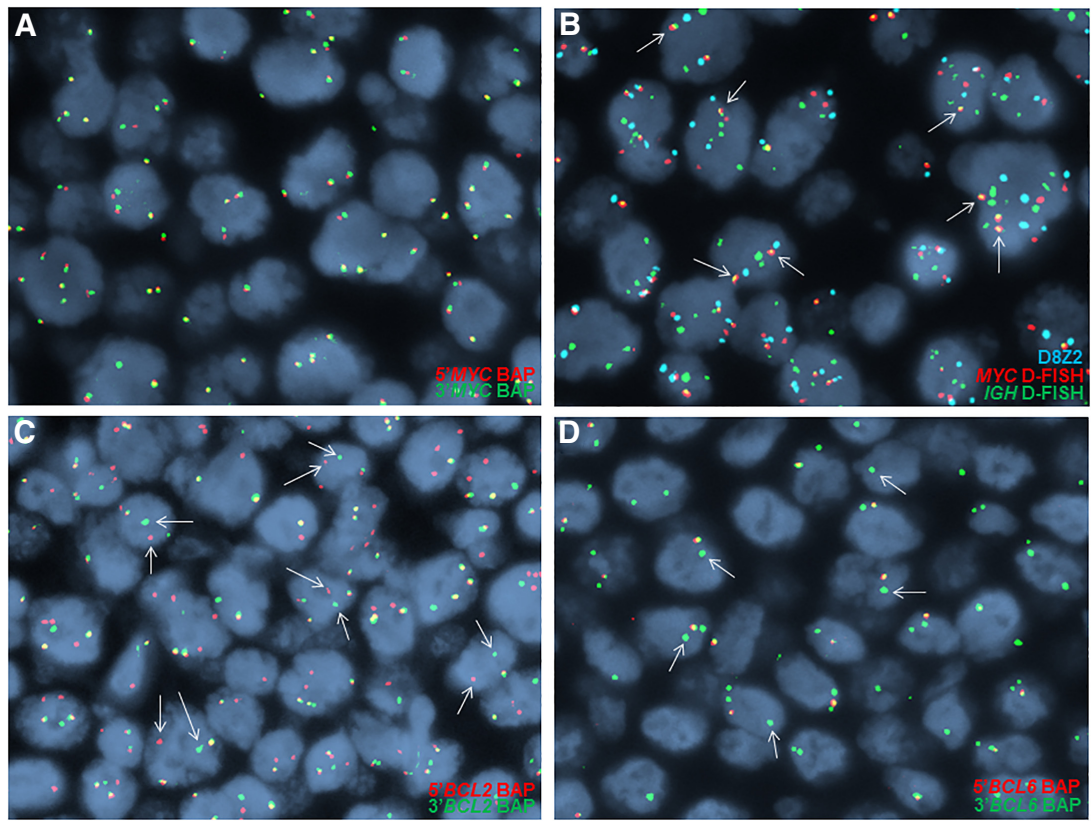

Figure 1. Representative fluorescence in situ hybridization (FISH) results for MYC BAP, IGH/MYC D-FISH, $B C L 2$ BAP, and BCL6 BAP studies performed on a paraffin-embedded left iliac lymph node specimen. (A) The MYC BAP was negative for MYC rearrangement, although additional intact MYC fusion signals were observed in 100\% of cells analyzed. (B) The IGH/MYC D-FISH revealed multiple fusions (arrows) representing a cryptic IGH/MYC rearrangement. (C) An apparently balanced BCL2 rearrangement indicated by split red and green signals (arrows). (D) An unbalanced BCL6 rearrangement indicated by loss of the $5^{\prime} B C L 6$ BAP (red) with retention of the $3^{\prime} B C L 6 B A P$, indicated by a single green signal (arrow).

Per our current laboratory algorithm to concurrently interrogate the MYC gene region with both MYC BAP and IGH/MYCD-FISH probe sets, we performed IGH/MYCD-FISH and identified an IGH/MYC fusion (abnormal cutoff $\geq 5$ ) (Fig. 1B). Although the BCL2 rearrangement appeared to be balanced (indicated by split red and green signals (Fig. 1C), the BCL6 rearrangement was interpreted as unbalanced as indicated by loss of the $5^{\prime}$ BCL6 BAP signal (red) (Fig. 1D).

To further characterize the discrepant MYC BAP (negative) and IGH/MYC D-FISH (positive) results, MPseq was performed and revealed an insertional rearrangement involving Chromosomes 8 and 14, resulting in a small segment ( $200 \mathrm{~kb})$ of the IGH locus from Chromosome 14 inserted downstream from the MYC gene on Chromosome 8 (Fig. 2A). Sanger sequencing was subsequently performed and confirmed the IGH/MYC rearrangement (Table 1). In addition, a t(14;18)(q32.33;q21.33) was identified by MPseq with breakpoints involving the IGH locus on Chromosome 14 and exon 3 of the BCL2 gene (NM_000633) on Chromosome 18 (Fig. 2B). Last, a segment of 3q27.3 harboring an intact $B C L 6$ gene was inserted into the 3 p24.3 chromosomal region, along with a heterozygous $3 q 27.3$ deletion that spanned the 5' BCL6 BAP footprint (Fig. 2C).

\section{DISCUSSION}

Both fresh and paraffin-embedded inguinal lymph node tissue involved by lymphoma from a 60-yr-old male patient were evaluated by hematopathology and laboratory genomics and a diagnosis of $\mathrm{HGBL}$ with $M Y C, B C L 2$, and BCL6 rearrangements was rendered. However, 

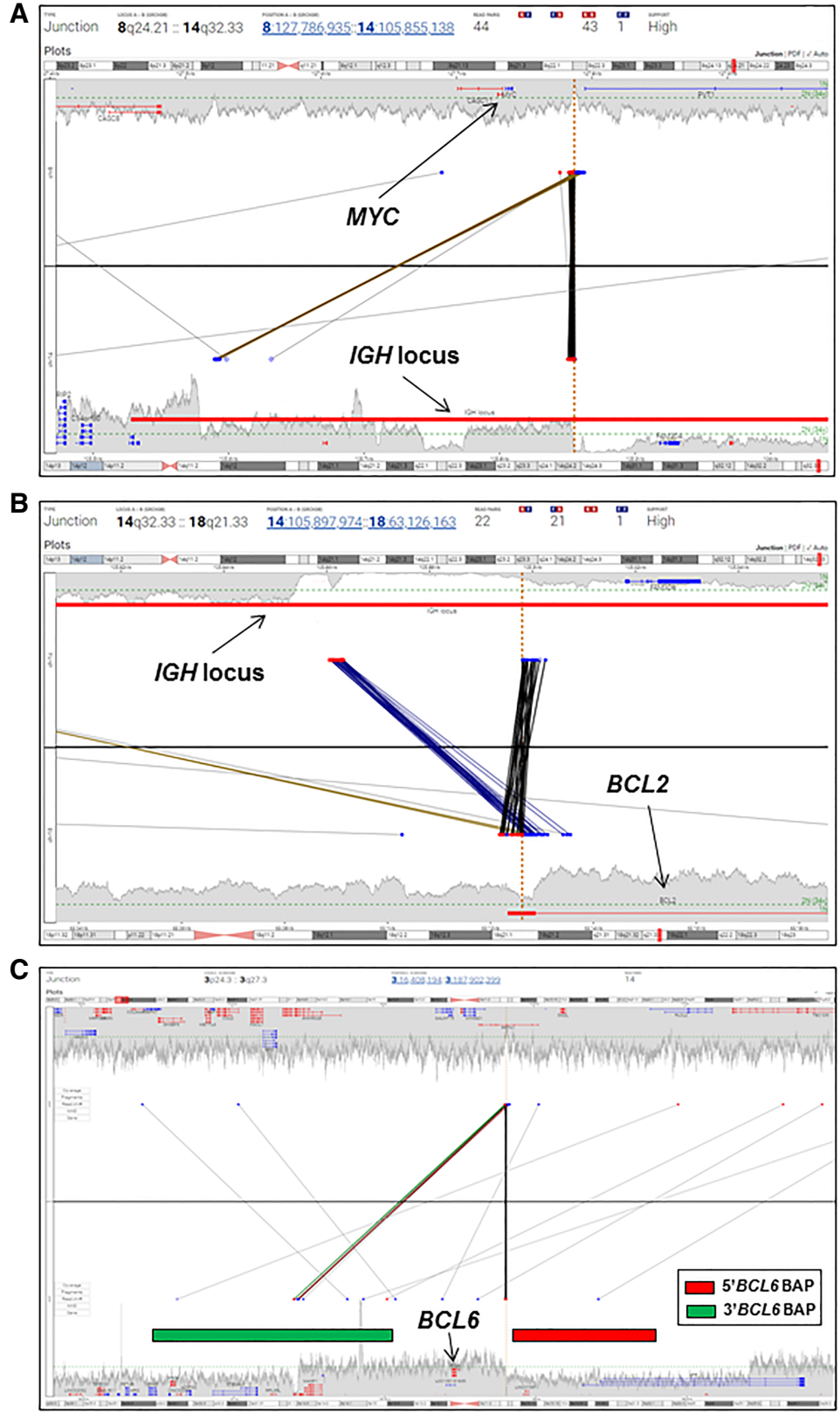

Figure 2. Mate-pair sequencing (MPseq) results. (A) Junction plot demonstrating a small segment ( 200 kb) of the IGH locus from Chromosome 14 inserted downstream from the MYC gene on Chromosome 8. (B) Junction plot demonstrating a rearrangement between the IGH locus on Chromosome 14 and exon 3 of the BCL2 gene (NM_000633) on Chromosome 18. (C) Junction plot demonstrating a 3q27.3 insertional event into the 3 p24.3 chromosomal region. In addition, a 3q27.3 deletion spanning the $5^{\prime}$ BCL6 BAP footprint was observed. Importantly, this insertional event does not disrupt or juxtapose BCL6 near immunoglobulin heavy or light chains

additional evaluation by MPseq indicated IGH/MYC and IGH/BCL2 rearrangements, in addition to a complex Chromosome 3 rearrangement of unclear clinical significance that did not disrupt or juxtapose BCL6 near immunoglobulin heavy or light chains (Fig. 3). 
Table 1. Mate-pair (MPseq) and Sanger sequencing results for the IGH/MYC rearrangement

\begin{tabular}{lllll}
\hline $\begin{array}{l}\text { MPseq event/ } \\
\text { position }\end{array}$ & Sanger breakpoints [hg38] & MPseq breakpoints [hg38] & Gene/locus & Primer sequence \\
\hline FF/position A & Chr 8:127,788,254 & Chr 8:127,788,231 & No gene & GCAACCCCGTCTCCACTAAA \\
FF/position B & Chr 14:105,589,660 & Chr 14:105,589,646 & IGH & ACTGGGTGAGCTTAGGTGGA \\
RR/position A & Chr 8:127,787,036 & Chr 14:127,786,935 & No gene & ACGGAAGTTTGCAAGCAAGA \\
RR/position B & Chr 14:105,855,473 & Chr 14:105,855,138 & IGH & AGATGGTCTGCTTCAGTGGC
\end{tabular}

Understanding the limitations of each MYCFISH probe set is critical as clinical cytogenetic laboratories routinely screen for MYC rearrangements in mature B-cell neoplasms with IGH/MYC D-FISH and/or MYC BAP sets. In our current case, MPseq accurately detected an insertion of the IGH locus downstream from the MYC gene region and was subsequently confirmed by Sanger sequencing. Although the IGH/MYC D-FISH probe set detected the IGH/MYC fusion, the MYC BAP demonstrated a false-negative result because the $\sim 200-\mathrm{kb}$ IGH insertion did not disrupt the $5^{\prime} M Y C$ and $3^{\prime}$ MYC BAP sufficiently to be appreciated by interphase cell analysis. Additional limitations of FISH are also highlighted by the BCL6 FISH result. Although the abnormal signal pattern represented an unbalanced $3 q 27.3$ rearrangement, MPseq clearly demonstrated that the BCL6 gene was not disrupted or juxtaposed near immunoglobulin heavy or light chains. This finding suggests that the $3 q 27.3$ rearrangement does not result in BCL6 overexpression as indicated by negative BCL6 IHC staining.

In conclusion, we fully characterized a novel IGH/MYC fusion by MPseq in a 60-yr-old male with HGBL that went undetected using a commercial MYC BAP set. Although MPseq also revealed the expected IGH/BCL2 rearrangement that was detected by FISH, a deletion spanning the 5' BCL6 BAP footprint was observed with unclear clinical

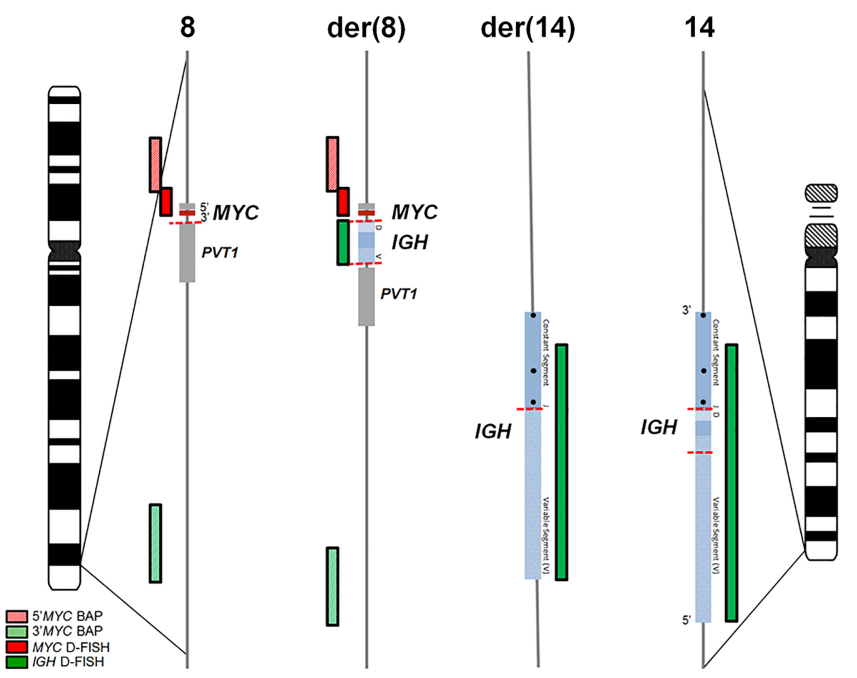

Figure 3. A focused view of the IGH and MYC gene regions on the derivative copies of Chromosomes 8 and 14. Horizontal dashed red lines indicate the breakpoints on derivative Chromosomes 8 and 14, and the subsequent location of the MYCBAP and IGH/MYCD-FISH footprints. The insertion of a small segment ( 200 kb) from the IGH locus downstream from MYC on Chromosome 8 produces a single fusion signal by IGH/MYC D-FISH studies. The minimal size of the insertional event precluded the detection of this rearrangement by MYC BAP studies. 
significance. Adding to the literature, this case provides additional evidence that all MYC rearrangements cannot be detected using a single commercially available MYC FISH probe set. Furthermore, this case highlights the power of MPseq to resolve structural and copy-number abnormalities beyond the capabilities of FISH. This observation may be more relevant in certain hematologic malignancies, including B- and T-cell lymphomas in which rearrangements driven by a positional effect are more common.

\section{METHODS}

\section{Fluorescence In Situ Hybridization (FISH)}

Fluorescence in situ hybridization (FISH) was performed on a FFPE left iliac lymph node specimen using commercially available (Abbott Molecular) D-FISH (IGH/MYC) and breakapart (BAP) (MYC, BCL2 and BCL6) probe sets. One hundred interphase nuclei were analyzed for each probe set. The FFPE specimen was subjected to standard FISH pretreatment, hybridization, and fluorescence microscopy according to specimen-specific protocols. FISH analysis was independently evaluated by two qualified clinical cytogenetic technologists and interpreted by a board-certified (ABMGG) clinical cytogeneticist.

\section{Mate-Pair Sequencing (MPseq)}

DNA was extracted from a fixed cell pellet (left iliac lymph node specimen) and $1 \mu \mathrm{g}$ was utilized for mate-pair sequencing library preparation and processed using the Illumina Nextera Mate Pair library kit (Illumina). Library preparation consisted of tagmentation to simultaneously shear and biotinylate the genomic DNA, strand displacement to fill any gaps left by the tagmentation step, and overnight circularization (16-20 h) to produce stabile 2-5 kb DNA fragments. AMPure purification (Beckman Coulter) was performed after the tagmentation and strand displacement steps $(0.56 \times$ and $0.4 \times$, respectively) to ensure only the longest fragments are selected to complete library preparation. After overnight circularization, noncircularized DNA was digested with exonuclease prior to mechanical shearing of the circularized fragments with a Covaris LE220 System (Covaris). The resulting biotinylated DNA fragments were bound to Dynabeads M-280 Streptavidin (Thermo Fisher Scientific) and subsequently processed through end repair, a-tailing, ligation of 7-bp Illumina adapters (a component of the TruSeq DNA library prep kit), and PCR using the PCR Primer Cocktail (Illumina) and KAPA HiFi HotStart Ready Mix PCR Kit (KAPA Biosystems). A $0.67 \times$ AMPure purification was performed to complete library preparation. MPseq libraries were multiplexed at two samples per lane to be sequenced on the Illumina HiSeq 2500 in rapid run mode. On both ends of each mate-pair fragment, 101 base pairs were sequenced to a bridged coverage of $43 \times$ and a base coverage of $\sim 6 \times$. Data were aligned to the reference genome (GRCh38) using BIMAv3, and abnormalities were identified and visualized using SVAtools, an in-house developed bioinformatics tool. Additional information regarding MPseq technology and bioinformatics tools have been previously described (Drucker et al. 2014; Johnson et al. 2018; Smadbeck et al. 2018).

\section{Sanger Sequencing}

Reference DNA sequences spanning the minimal 5' and maximal 3' positions of MPseq approximate breakpoints were used for primer design using Primer3Plus. End point PCR was performed on patient DNA with a 50\% 2X Paq5000 Hotstart PCR Master Mix (Agilent) using touchdown PCR. Results were visualized on a $2 \%$ agarose gel in a UV light box, and amplicon sizes were estimated. Selected amplicons were purified with Exo-SAP-IT PCR Product Cleanup Reagent (Thermo Fisher Scientific) and Sanger sequencing was performed on a 
Competing Interest Statement Algorithms described in this manuscript for mate-pair sequencing are licensed to WholeGenome LLC, which is owned by G.V.

\section{Referees}

Ryan D. Morin

Pedro Farinha

Anonymous

Received February 18, 2019 accepted in revised form March 27, 2019. 3730x/ DNA Analyzer (Thermo Fisher Scientific). The resulting sequences were analyzed using Sequencher DNA Sequence Analysis Software (Gene Codes Corporation) and mapped to the GRCh38 genome using the BLAT function in the UCSC genome browser to determine precise breakpoints in this rearrangement.

\section{ADDITIONAL INFORMATION}

\section{Data Deposition and Access}

The variants were submitted to ClinVar (http://www.ncbi.nlm.nih.gov/clinvar/) and can be found under accession numbers SCV000902268.1 and SCV000902269.1.

\section{Ethics Statement}

The results described in this manuscript were derived from clinical rather than research testing. Because patient identifiers were removed for the purposes of this manuscript, consent (oral or written) was not required and Mayo Clinic does not require IRB approval for the publication of single case reports.

\section{Author Contributions}

B.A.P., J.F.P., and L.B.B. drafted and edited the manuscript, analyzed data, and generated figures. S.A.S. analyzed data, generated figures, and reviewed and edited the manuscript. G.V., J.B.S., P.T.G., and R.P.K. reviewed and edited the manuscript. W.R.M. collected and analyzed data and reviewed and edited the manuscript

\section{Funding}

Funding for this project was provided by the Mayo Clinic Department of Laboratory Medicine and Pathology with no external or National Institutes of Health funding used.

\section{REFERENCES}

Drucker TM, Johnson SH, Murphy SJ, Cradic KW, Thereau TM, Vasmatzis G. 2014. BIMA V3: an aligner customized for mate pair library sequencing. Bioinformatics 30: 1627-1629. doi:10.1093/bioinformatics/ btu078

Johnson SH, Smadbeck JB, Smoley SA, Gaitatzes A, Murphy SJ, Harris FR, Drucker TM, Zenka RM, Pitel BA, Rowsey RA, et al. 2018. SVAtools for junction detection of genome-wide chromosomal rearrangements by mate-pair sequencing (MPseq). Cancer Genet 221: 1-18. doi:10.1016/j.cancergen.2017.11.009

King RL, McPhail ED, Meyer RG, Vasmatzis G, Pearce K, Smadbeck JB, Ketterling RP, Smoley SA, Greipp PT, Hoppman NL, et al. 2018. False-negative rates for MYC FISH probes in B-cell neoplasms. Haematologica doi:10.3324/haematol.2018.207290.

Kluin PM, Harris NL, Stein H, Leoncini L, Campo E, Jaffe ES, Gascoyne RD, Swerdlow SH. 2017. High-grade Bcell lymphoma. In WHO classification of tumours of haematopoietic and lymphoid tissues, 4th ed. (ed. Swerdlow SH, et al.), pp. 335-341. IARC, Lyon, France.

Li S, Lin P, Fayad LE, Lennon PA, Miranda RN, Yin CC, Lin E, Medeiros LJ. 2012. B-cell lymphomas with MYC/ $8 q 24$ rearrangements and IGH@BCL2/t(14;18) (q32;q21): an aggressive disease with heterogeneous histology, germinal center B-cell immunophenotype and poor outcome. Mod Pathol 25: 145-156. doi:10 .1038/modpathol.2011.147

Li S, Desai P, Lin P, Yin CC, Tang G, Wang XJ, Konoplev SN, Khoury JD, Bueso-Ramos CE, Medeiros LJ. 2016. MYC/BCL6 double-hit lymphoma (DHL): a tumour associated with an aggressive clinical course and poor prognosis. Histopathology 68: 1090-1098. doi:10.1111/his.12884

May PC, Foot N, Dunn R, Geoghegan H, Neat MJ. 2010. Detection of cryptic and variant IGH-MYC rearrangements in high-grade non-Hodgkin's lymphoma by fluorescence in situ hybridization: implications for cytogenetic testing. Cancer Genet Cytogenet 198: 71-75. doi:10.1016/j.cancergencyto.2009.12.010 
Muñoz-Mármol AM, Sanz C, Tapia G, Marginet R, Ariza A, Mate JL. 2013. MYC status determination in aggressive B-cell lymphoma: the impact of FISH probe selection. Histopathology 63: 418-424. doi:10.1111/his .12178

Sesques $P$, Johnson NA. 2017. Approach to the diagnosis and treatment of high-grade B-cell lymphomas with MYC and BCL2 and/or BCL6 rearrangements. Blood 129: 280-288. doi:10.1182/blood-2016-02-636316

Smadbeck JB, Johnson SH, Smoley SA, Gaitatzes A, Drucker TM, Zenka RM, Kosari F, Murphy SJ, Hoppman N et al. 2018. Copy number variant analysis using genome-wide mate-pair sequencing. Genes Chromosomes Cancer 57: 459-470. doi:10.1002/gcc.5

Snuderl M, Kolman OK, Chen YB, Hsu JJ, Ackerman AM, Dal Cin P, Ferry JA, Harris NL, Hasserjian RP, Zukerberg LR, et al. 2010. B-cell lymphomas with concurrent IGH-BCL2 and MYC rearrangements are aggressive neoplasms with clinical and pathologic features distinct from Burkitt lymphoma and diffuse large B-cell lymphoma. Am J Surg Pathol 34: 327-340. doi:10.1097/PAS.0b013e3181cd3aeb

Sun G, Montella L, Yang M. 2012. MYC gene FISH testing in aggressive B-cell lymphomas: atypical rearrangements may result in underreporting of positive cases. Blood 120: 1552. doi:10.1182/blood-2012-04408724

Wang W, Hu S, Lu X, Young KH, Medeiros LJ. 2015. Triple-hit B-cell lymphoma with MYC, BCL2, and BCL6 translocation/rearrangements: clinicopathologic features of 11 cases. Am J Surg Pathol 39: 1132-1139. doi:10.1097/PAS.0000000000000434 


\section{COLD SPRING HARBOR Molecular Case Studies}

\section{Elucidating a false-negative $M Y C$ break-apart fluorescence in situ hybridization probe study by next-generation sequencing in a patient with high-grade B-cell lymphoma with IGH/MYC and $I G H / B C L 2$ rearrangements}

Jess F. Peterson, Beth A. Pitel, Stephanie A. Smoley, et al.

Cold Spring Harb Mol Case Stud 2019, 5: a004077

Access the most recent version at doi: $10.1101 /$ mcs.a004077

References This article cites 12 articles, 3 of which can be accessed free at: http://molecularcasestudies.cshlp.org/content/5/3/a004077.full.html\#ref-list-1

License This article is distributed under the terms of the Creative Commons

Attribution-NonCommercial License, which permits reuse and redistribution, except for commercial purposes, provided that the original author and source are credited.

Email Alerting Receive free email alerts when new articles cite this article - sign up in the box at the Service top right corner of the article or click here. 